\title{
Clinical Utility of Pegaspargase in Children, Adolescents and Young Adult Patients with Acute Lymphoblastic Leukemia: A Review
}

\author{
Cynthia Bender' \\ Luke Maese ${ }^{2}$ \\ Maria Carter-Febres ${ }^{2}$ \\ Anupam Verma $\mathbb{D}^{2}$ \\ 'Department of Pharmacy, Primary \\ Children's Hospital, Salt Lake City, UT, \\ USA; ${ }^{2}$ Division of Hematology/Oncology, \\ Department of Pediatrics, University of \\ Utah and Primary Children's Hospital, \\ Salt Lake City, UT, USA
}

Correspondence: Anupam Verma Pediatric Hematology/Oncology, University of Utah, Primary Children's Hospital, 100 N Mario Capecchi Drive, Salt Lake City, UT, 84I I3, USA

Tel + I 8016624700

Fax +I 8016624707

Email anupam.verma@hsc.utah.edu

\begin{abstract}
Acute lymphoblastic leukemia (ALL) is a heterogenous hematological malignancy representing $25 \%$ of all cancers in children less than 15 years of age. Significant improvements in survival and cure rates have been made over the past four decades in pediatric ALL treatment. Asparaginases, derived from Escherichia coli and Erwinia chrysanthemi, have become a critical component of ALL therapy since the 1960s. Asparaginases cause depletion of serum asparagine, leading to deprivation of this critical amino acid for protein synthesis, and hence limit survival of lymphoblasts. Pegaspargase, a conjugate of monomethoxypolyethylene glycol (mPEG) and L-asparaginase, has become an integral component of pediatric upfront and relapsed ALL protocols due to its longer half-life and improved immunogenicity profile compared to native asparaginase preparations. Over the past two decades great strides have been made in outcomes for pediatric ALL due to risk stratification, incorporation of multiagent chemotherapy protocols, and central nervous system prophylaxis with pegaspargase having played an important role in this success. However, adolescents and young adults (AYA) with ALL when treated on contemporaneous trials using adult ALL regimens, continue to have poor outcomes. There is increasing realization of adapting pediatric trial regimens for treating AYAs, especially those incorporating higher intensity of chemotherapeutic agents with pegaspargase being one such agent. Dose or treatment-limiting toxicity is observed in $25-30 \%$ of patients, most notable being hypersensitivity reactions. Other toxicities include asparaginase-associated pancreatitis, thrombosis, liver dysfunction, osteonecrosis, and dyslipidemia. Discontinuation or subtherapeutic levels of asparaginase are associated with inferior disease-free survival leading to higher risk of relapse, and in cases of relapse, a higher risk for remission failure. This article provides an overview of available evidence for use of pegaspargase in pediatric acute lymphoblastic leukemia.
\end{abstract}

Keywords: asparagine, asparaginase, serum asparaginase activity, toxicity

\section{Introduction}

Acute lymphoblastic leukemia (ALL) is the most commonly encountered cancer of childhood with peak prevalence between ages 2-5 years. Two main subtypes, B-cell and T-cell, comprise the majority of ALL, at $85-90 \%$ and $10-15 \%$ of cases, respectively. ${ }^{1}$ Outcomes within pediatric ALL vary by subtype and risk category, but are generally favorable. While within standard-risk (SR) B-cell ALL overall survival (OS) is as high as $96 \%$, high risk (HR) B-ALL and T-ALL 
continue to have lower event-free survival (EFS) and OS. $^{2-6}$ Over the past several decades, significant progress has been made with risk stratification and chemotherapy dose intensification. Asparaginase, introduced as a component of the chemotherapeutic backbone for ALL in the 1970s, has been of significant importance in advancing the treatment of childhood leukemia. ${ }^{3,7,8}$ Its antileukemic activity is accomplished by converting asparagine to aspartic acid and ammonia in the extracellular fluid. Lymphoblasts are very sensitive to the lack of asparagine and this shortage reduces the synthesis of asparagine-dependent proteins causing cell death. ${ }^{9}$ There are extensive clinical data supporting the use and improved survival benefits of asparaginase therapy in pediatric ALL. ${ }^{2,3,10-17}$ Omission or low therapeutic levels of asparaginase may lead to higher risk of relapse, and in cases of relapse, a higher risk for remission failure. ${ }^{3,18-20}$ Pegaspargase $\left(\right.$ Oncaspar $^{\circledR}$ ), a pegylated form of native Escherichia coli (E. coli) derived L-asparaginase, is the preferred first-line asparaginase preparation in the multiagent chemotherapy regimens for treatment of childhood ALL in the United States (US) and European Union (EU). ${ }^{21-23} \mathrm{We}$ provide an overview on the use of pegaspargase in pediatric ALL.

\section{Pharmacological Characteristics of Pegaspargase}

In comparison to healthy cells, leukemic cells have low levels of asparagine synthetase and hence, reduced ability to manufacture the essential amino acid asparagine needed for DNA, RNA and protein synthesis. ${ }^{24,25}$ Asparaginase hydrolyzes asparagine into aspartic acid and ammonia in the plasma, thus denying the lymphoblast an essential element needed for cell survival. ${ }^{26}$ Asparaginase was identified as a potential chemotherapeutic in 1961 when it was first isolated as an antilymphoma component of guinea pig serum. ${ }^{27}$ Studies in the 1960s used bacteria as alternate sources of asparaginase, leading to clinically available asparaginase derived from E. coli and Erwinia chrysanthemi. ${ }^{28,29}$

Pegaspargase is a conjugate of monomethoxypolyethylene glycol (mPEG) and L-asparaginase produced endogenously by $E$. coli. These mPEG molecules increase solubility and stability of the protein conjugate in the plasma and prevent proteolytic cleavage of the enzyme, consequently increasing its half-life. $^{22,30}$ They also provide an additional benefit of reduction in the risk of anaphylaxis and hypersensitivity reactions.

\section{Dosage and Administration}

Pegaspargase is supplied as a sterile, preservative-free solution in vials containing 3750 International Units (IU) of pegaspargase per $5 \mathrm{~mL}$ solution. $^{21,22}$ The FDAapproved dose for pediatric leukemia is $2500 \mathrm{IU} / \mathrm{m}^{2}$ given either intramuscularly (IM) or intravenously (IV) over 1-2 hours, not more often than every 14 days. ${ }^{21,22}$ Increased toxicity due to higher doses of pegaspargase is controversial. Lebovic et al reported a retrospective chart review of patients 1-21 years of age with B-ALL on pegaspargase and found that doses higher than $3750 \mathrm{IU}$ were associated with higher rates of venous thromboembolism (VTE), pancreatitis, and hyperglycemia. ${ }^{31}$ Dose capping is frequently employed in the adult population, particularly in obese patients; however, it has not been standard practice in pediatrics and further studies should investigate the potential for toxicity in this subset of patients.

\section{Pharmacokinetic (PK) Comparison Between IV and IM Administration}

The goal of asparaginase therapy is to achieve complete asparagine depletion. Quantification of asparagine directly is biologically and logistically challenging, particularly in the clinical setting, so serum asparaginase activity (SAA) levels are accepted as the most reliable modality to measure asparaginase efficacy. ${ }^{32}$ While there has been recent debate regarding optimal SAA levels for adequate asparagine depletion, an SAA of $>0.1 \mathrm{IU} / \mathrm{mL}$ is generally considered optimal. ${ }^{24,32-35}$ Both Children's Cancer Group (CCG) 1962 and Children's Oncology Group (COG) AALL07P4 studies have provided important PK data on IM and IV administration, respectively. ${ }^{24,34}$ After an IM dose of 2500 $\mathrm{IU} / \mathrm{m}^{2}$, the maximum concentration of asparaginase activity peaked at $1 \mathrm{IU} / \mathrm{mL}$ compared to $1.6 \mathrm{IU} / \mathrm{mL}$ for IV on day 5 with a half-life absorption estimated at 1.7 days. ${ }^{21,22,24}$ The replacement of native asparaginase with pegaspargase in the treatment of pediatric ALL has come about due to its prolonged terminal half-life. The average half-life is estimated at 5.5 days for both IM and IV administration and is $4-8$ 
times longer than those of the native and Erwiniaderived products. ${ }^{22,36}$ Silverman et al also confirmed that enzyme activity is maintained for a minimum of 2 weeks after a single IV dose, thus supporting the recommendation that dosing not occur more frequently than 2-week intervals. ${ }^{22,37}$

\section{Comparison with Other Pegylated Products}

Calaspargase (CALASP) was developed using the same asparaginase enzyme and PEG molecule as pegaspargase. However, whereas pegaspargase has a succinimidyl succinate (SS-PEG) molecule which combines the two, CALASP consists instead of a succinimidyl carbonate (SC-PEG) linkage which enables greater biologic stability. This stability is realized in the much longer shelf life of CALASP, 36 months, versus pegaspargase at 8 months. COG AALL07P4 investigated the PK and pharmacodynamic (PD) properties of calaspargase pegol (Asparlas $\left.{ }^{\circledR}\right){ }^{38}$ The results demonstrated a potential advantage to its use with CALASP having a more prolonged SAA compared with pegaspargase through both induction and consolidation. ${ }^{38}$ The mean half-life SAA was 2.5 times longer with CALASP than with pegaspargase, representing a more prolonged suppression of asparagine in the plasma. ${ }^{38}$ Although not statistically significant, adverse effects were similar between both groups other than CALASP had increased hyperglycemia in induction and hyperbilirubinemia in delayed intensification (DI). ${ }^{38}$ Dana-Farber Cancer Institute (DFCI) 11-001 randomized patients to either pegaspargase every 2 weeks or CALASP every 3 weeks during intensification (Table 1). There were no significant differences in treatment outcomes or toxicities between the two arms, but PK data showed more prolonged SAA after CALASP in the induction phase. ${ }^{39}$ As the DFCI protocols have 3 -week cycles during intensification, CALASP is an ideal fit for the asparagine depletion prescribed in their chemotherapeutic backbone. This new pegylated product, as well as the yet unproven pegylated Erwinia asparaginase (pegcrisantaspase), may offer future alternate treatment options to pegaspargase. $^{38,40,41}$

Table I Pediatric Acute Lymphoblastic Leukemia Trials with Pegaspargase Associated Objectives

\begin{tabular}{|c|c|c|c|}
\hline $\begin{array}{l}\text { Cooperative Group/ } \\
\text { Study }\end{array}$ & $\begin{array}{l}\text { Enrollment Period } \\
\text { (N) }\end{array}$ & $\begin{array}{l}\text { Asparaginase } \\
\text { Objective(s) }\end{array}$ & Asparaginase Associated Findings \\
\hline \multicolumn{4}{|l|}{ Upfront studies } \\
\hline $\mathrm{DFCl} 9 \mathrm{I}-0 \mathrm{I}^{3}$ & 199|-|995 (377) & $\begin{array}{l}\text { ASP intensification } \\
\text { PEG (IM) vs. L-asp (IM) }\end{array}$ & $\begin{array}{l}\text { - Intensification improves outcomes } \\
\text { - No significant difference in EFS with ASP preparation }\end{array}$ \\
\hline CCG $1962^{24}$ & 1997-1998 (187) & PEG (IM) vs. L-asp (IM) & - PEG: increased clearance of blasts, decreased antibodies \\
\hline AIEOP ALL $2000^{50}$ & $2002(20)$ & $\begin{array}{l}\text { PEG (IV) as first line } \\
\text { therapy }\end{array}$ & - Adequate SAA and ASN depletion \\
\hline DFCl $05-001^{33}$ & $2005-2010(463)$ & PEG (IV) vs. L-asp (IM) & $\begin{array}{l}\text { - PEG: increased ASP course completion success and } \\
\text { increased SAA } \\
\text { - No significant difference in DFS with ASP preparation }\end{array}$ \\
\hline COG AALL07P4 $4^{34,38}$ & $2008-2010(166)$ & PEG (IV) vs. CALASP (IV) & - CALASP: prolonged SAA \\
\hline DFCI II-001 $1^{39}$ & $2012-2015(230)$ & PEG (IV) vs. CALASP (IV) & - CALASP: prolonged SAA \\
\hline \multicolumn{4}{|l|}{ Relapsed studies } \\
\hline CCG $\left.194\right|^{18}$ & 1995-1998 (93) & PEG $(\mathrm{IM})$ in relapse & - Decreased ASN led to increased second remission \\
\hline CHMC $1001^{51}$ & $|997-200|$ (28) & PEG (IM) in relapse & - Adequate SAA and ASN depletion \\
\hline
\end{tabular}

Abbreviations: DFCl, Dana-Farber Cancer Institute; CCG, Children's Cancer Group; AIEOP, Associazione Italiana di Ematologia e Oncologia Pediatrica; COG, Children's Oncology Group; CHMC, Children's Hospital and Regional Medical Center; PEG, pegaspargase; L-asp, L-asparaginase; CALASP, calaspargase; ASP, asparaginase; IM, intramuscular; IV, intravenous; EFS, event-free survival; DFS, disease-free survival; SAA, serum asparaginase activity level; ASN, asparagine. 


\section{Pegaspargase in Newly Diagnosed ALL}

In the US there are different types of commercially available asparaginase formulations, an E. coli-derived pegylated asparaginase (pegaspargase) and an Erwinia derived asparaginase. The native E. coli derived L-asparaginase was removed from the US market by the manufacturer in 2012 but still plays an important historical role. The pegylated form of the drug, approved in 1994 for use in patients with ALL, increases the plasma retention time, decreases proteolysis and renal excretion, and protects from immune detection.

\section{Standard Risk (SR) ALL}

CCG 1962 was a randomized trial to evaluate safety, efficacy, and PK of a single IM dose of pegaspargase versus multiple IM doses of native $E$. coli asparaginase in each of 3 phases: a 4 -week induction and 2, 8-week DI phases as part of a multiagent chemotherapeutic regimen. ${ }^{24}$ One hundred eighteen children in 8 CCG centers with SR ALL were enrolled between 1997-1998. Patients were randomly assigned to receive either $2500 \mathrm{IU} / \mathrm{m}^{2}$ of pegaspargase $\mathrm{IM}$ on day 3 of induction and each DI phase, or $6000 \mathrm{IU} / \mathrm{m}^{2}$ of native asparaginase IM 3 times per week for 9 doses in induction and 6 doses in each DI phase (Table 1). Patients assigned to the pegaspargase arm had significantly more rapid clearance of lymphoblasts from days 7 and 14 bone marrow aspirates $(\mathrm{p} \leq 0.05)$. Twice $(\mathrm{n}=$ 16) as many patients in the native asparaginase arm had M3 bone marrow ( $>25 \%$ blasts) on day 7 than in the pegaspargase arm $(n=8)$. Four patients had M3 marrow on day 14 , all were in the native asparaginase arm. The study postulated that the difference could be from more persistent, higher SAA in the pegaspargase patients. ${ }^{24}$ Half-lives of asparaginase were 5.5 days and 26 hours for pegaspargase and native asparaginase, respectively. Studies have shown when antibody titers are high, the asparaginase activity is often low. ${ }^{42}$ Low SAA in the native arm (26\%) compared to the pegaspargase arm $(2 \%)$ during the first DI phase established the primary endpoint of this study that incidence of high anti-asparaginase antibody titers in children treated with pegaspargase would be decreased by at least $50 \%$ in the first DI compared with those treated with native asparaginase. Three-year EFS was similar for both arms, $85 \%$ for pegaspargase and $78 \%$ for native asparaginase. $^{24}$

\section{High Risk (HR) ALL}

DCFI 91-01 protocol was designed to improve the outcome of children with newly diagnosed ALL while minimizing toxicity. ${ }^{3}$ Three hundred and seventy-seven patients (137 SR and 240 HR), aged 0-18 years, were enrolled between 1991-1995. ${ }^{3}$ Apart from substituting dexamethasone for prednisone, the duration of high dose asparaginase intensification was extended from 20 weeks (in prior protocols) to 30 weeks, and patients were randomized to receive either native or pegylated E. coli asparaginase (Table 1). Patients were administered either $2500 \mathrm{IU} / \mathrm{m}^{2}$ pegaspargase IM every other week for 15 doses or $25,000 \mathrm{IU} / \mathrm{m}^{2}$ native $E$. coli asparaginase IM every week for 30 doses during the intensification phase of therapy. Though the study did not find a significant difference in 5-year EFS based upon risk group $(87 \% \pm 3 \%$ for SR and $81 \% \pm 3 \%$ for HR, $\mathrm{p}=$ 0.24 ), asparaginase intolerance (failure to receive at least 26 weeks of asparaginase) was an independent predictor of adverse outcome in multivariate analysis $(\mathrm{p}<0.01)$. This finding suggested that the prolongation of asparaginase therapy may have contributed to the improved overall outcome of 5 -year EFS $(83 \% \pm 2 \%)$ compared to previous protocols. ${ }^{3}$

DFCI 05-001, conducted between 2005-2010, enrolled 551 newly diagnosed ALL patients aged 1-18 years, with the aim to compare the relative toxicity and efficacy of IV pegaspargase and IM native E. coli L-asparaginase in both SR and HR ALL patients. $^{33}$ Those patients who achieved complete remission (CR) after induction therapy were assigned to a final risk group and randomized to IV pegaspargase (15 doses of $2500 \mathrm{IU} / \mathrm{m}^{2}$ every 2 weeks) or IM native L-asparaginase (30 doses of $25,000 \mathrm{IU} / \mathrm{m}^{2}$ weekly), beginning at week 7 after study entry (Table 1). The primary endpoint of the study was to evaluate asparaginase-related toxicities, while EFS, SAA, and quality of life during therapy as assessed by PedsQL surveys were secondary endpoints. The two treatme $7 \mathrm{nt}$ groups did not have statistically significant difference in overall frequency of asparaginase-related toxicities; $28 \%$ in the IV pegaspargase group versus $26 \%$ in IM native L-asparaginase group $(\mathrm{p}=0.60)$. A significantly higher proportion of pegaspargase than native $L$-asparaginase recipients completed all 30 weeks of 
treatment ( 82 vs. $74 \% ; p=0.015)$. The median nadir SAA of $\geq 0.1 \mathrm{IU} / \mathrm{mL}$ was significantly higher in patients who received IV pegaspargase than in those who received IM native L-asparaginase (99 vs. 71\%; p < $0.0001){ }^{33}$ Significantly more anxiety was reported by both patients and parent-proxy in the IM native L-asparaginase group than in the IV pegaspargase group ( $\mathrm{p} \leq 0.03$ ). With a median follow-up of 6 years, 5-year EFS was $90 \%$ for IV pegaspargase versus $89 \%$ for IM native L-asparaginase $(p=0.58) .^{33}$

Pediatric Oncology Group (POG) study POG 8704 (T-3) established in 357 patients with T ALL and 195 patients with advanced-stage $\mathrm{T}$ lymphoblastic lymphoma (LLy) that high dose asparaginase consolidation therapy improves survival in pediatric patients. ${ }^{13}$

Associazione Italiana di Ematologia e Oncologia Pediatrica (AIEOP) ALL 2000 protocol, utilizing an intensive multiagent Berlin-Frankfurt-Munster (BFM) chemotherapy backbone, studied the pharmacological effects of pegaspargase as the first-line product in children with ALL. ${ }^{43}$ Twenty patients with SR, intermediate-risk (IR), and HR ALL on the protocol were given 2 doses and 1 dose of pegaspargase during induction and reinduction phases, respectively (Table 1). The investigators studied the asparagine depletion and clinical outcomes against a comparison cohort of 37 patients treated with the same chemotherapy schedule but with native asparaginase. This study demonstrated pegaspargase effectively substituted for the native asparaginase product in regard to serum activity levels and asparagine depletion. Additionally, allergic reactions and immunologically mediated silent inactivation were drastically minimized despite multiple exposures to the drug. 43

\section{Adolescents and Young Adults (AYA) ALL} Survival rates in AYA ALL are poor compared with those in younger children. Surveillance epidemiology and end results (SEER) data from 2000-2004 in ALL, reported a 10 -year OS of $80 \%$ in children $<15$ years, falling to $60 \%$ in adolescents aged 15-20 years, and 30\% in young adults aged 20-30 years. ${ }^{44,45}$ Multiple studies have looked at adopting pediatric protocols for AYA with improved overall outcomes. Asparaginase, as it does in younger children, plays an important role in AYA ALL therapy. Ram et al conducted a systematic review and metaanalysis of all comparative trials on AYA patients given chemotherapy with either pediatric-inspired regimens or conventional adult protocols, reviewing 11 trials and 2489 patients. $^{46}$ AYA patients given pediatric inspired regimens had a statistically significant lower mortality rate at 3 years (RR $0.58 ; 95 \%$ CI $0.51-0.67$ ), lower relapse rate (RR 0.51; 95\% CI 0.39-0.66), superior CR (RR 1.05; 95\% CI 1.01-1.10) and EFS (RR 1.66; 95\% CI 1.39-1.99). ${ }^{46}$ The German Multicenter Study Group For Adult ALL (GMALL) protocols were originally based on pediatric BFM protocols and designed for AYA ALL. ${ }^{47}$ GMALL 07/03 enrolled 877 patients, utilized intensified shortened induction with dexamethasone rather than with prednisone, pegaspargase rather than native asparaginase in induction and consolidation, plus incorporated changes in the management of minimal residual disease with stem cell transplant. GMALL 07/03 had significant improvements in 5-year OS compared with previous protocol GMALL $05 / 93$ (65\% in GMALL $07 / 03$ vs $46 \%$ in GMALL 05/93). ${ }^{47}$ A French study examined the outcomes of 177 AYAs aged 15-20 years comparing those who were treated on a pediatric inspired protocol FRALLE-93, or an adult protocol LALA-94A. ${ }^{48}$ FRALLE-93 showed significant better CR $(98 \%$ v $81 \% ; \mathrm{p}=0.002)$ and EFS ( $\mathrm{p}$ $=0.0002)$ in B-ALL, and EFS $(p=0.05)$ in T-ALL. The cumulative doses of chemotherapies, most notably asparaginase, were higher in the pediatric protocol: 180,000 IU/ $\mathrm{m}^{2}$ in the pediatric regimen versus $9000 \mathrm{IU} / \mathrm{m}^{2}$ in the adult regimen. ${ }^{48}$ Italian and Dutch retrospective studies compared outcomes in adolescents aged 14-18 years treated on pediatric AIEOP ALL 95 and 2000 protocols with those treated on the adult Gruppo Italiano per le Malattie Ematologiche dell'Adulto (GIMEMA) ALL 0496 and 2000 protocols, and Dutch Children's Oncology Group (DCOG) pediatric regimen with the Hemato-Oncologie voor Volwassenen Nederland (HOVON) adult protocols in AYAs. Both comparisons showed improved outcomes in patients treated on pediatric protocols. The primary differences between the regimens were shorter intervals between courses ( $\leq 1$ week versus $\leq 4$ weeks) and more asparaginase (mean cumulative dosage: 101,000 IU/m ${ }^{2}$ vs. $70,000 \mathrm{IU} / \mathrm{m}^{2}$ ) in the pediatric regimens. ${ }^{49,50}$

Pediatric inspired regimens use higher doses of chemotherapeutic agents at shorter intervals, including pegaspargase, which have demonstrated superiority to conventional adult regimens in AYA ALL patients. Dosing scheme is not the only reason for improved outcomes as there are biological differences in AYAs that also play a role. Not only do AYAs have more toxicity to the intensive chemotherapies, they also have 
Table 2 Standard Risk B-ALL Regimen (Adapted from Children's Oncology Group AALL0932)

\begin{tabular}{|c|c|c|}
\hline Phase & $\begin{array}{l}\text { Chemotherapy } \\
\text { Agents }\end{array}$ & Schedule \\
\hline $\begin{array}{l}\text { Induction } \\
\qquad \text { (35 days) }\end{array}$ & $\begin{array}{l}\text { Dexamethasone PO } \\
\text { Vincristine IV } \\
\text { Pegaspargase IV } \\
\text { Cytarabine IT } \\
\text { Methotrexate IT }\end{array}$ & $\begin{array}{l}\text { Days I- } 28 \\
\text { Days I, } 8,15,22 \\
\text { Day } 4 \\
\text { Day I (CNS2 require more) } \\
\text { Days } 8,29\end{array}$ \\
\hline $\begin{array}{l}\text { Consolidation } \\
\text { ( } 28 \text { days) }\end{array}$ & $\begin{array}{l}\text { Mercaptopurine PO } \\
\text { Vincristine IV } \\
\text { Methotrexate IT }\end{array}$ & $\begin{array}{l}\text { Days I-28 } \\
\text { Day I } \\
\text { Days I, 8, I5 }\end{array}$ \\
\hline $\begin{array}{l}\text { Interim } \\
\text { Maintenance } \\
\text { (IM I) } \\
\quad \text { (56 days) }\end{array}$ & $\begin{array}{l}\text { Vincristine IV } \\
\text { Methotrexate IV } \\
\text { (escalating dose) } \\
\text { Methotrexate IT }\end{array}$ & $\begin{array}{l}\text { Days I, II, 2I, 3I, 4I } \\
\text { Days I, II, 2I, 3I, } 4 \text { I } \\
\text { Day 3I }\end{array}$ \\
\hline $\begin{array}{l}\text { Delayed } \\
\text { Intensification } \\
\qquad \text { (56 days) }\end{array}$ & $\begin{array}{l}\text { Dexamethasone PO } \\
\text { Thioguanine PO } \\
\text { Vincristine IV } \\
\text { Doxorubicin IV } \\
\text { Pegaspargase IV } \\
\text { Cyclophosphamide IV } \\
\text { Cytarabine IV } \\
\text { Methotrexate IT }\end{array}$ & $\begin{array}{l}\text { Days } 1-7 \text { and } 15-2 \mid \\
\text { Days } 29-42 \\
\text { Days I, 8, } 15 \\
\text { Days I, 8, } 15 \\
\text { Day } 4 \\
\text { Day } 29 \\
\text { Days } 29-32 \text { and 36-39 } \\
\text { Days I, } 29\end{array}$ \\
\hline $\begin{array}{l}\text { Interim } \\
\text { Maintenance } \\
\text { (IM 2) } \\
\text { (56 days) }\end{array}$ & $\begin{array}{l}\text { Vincristine IV } \\
\text { Methotrexate IV } \\
\text { (escalating dose) } \\
\text { Methotrexate IT }\end{array}$ & $\begin{array}{l}\text { Days I, II, 2I, 3I, 4I } \\
\text { Days I, II, 2I, 3I, } 4 \text { I } \\
\text { Days I, 3I }\end{array}$ \\
\hline $\begin{array}{l}\text { Maintenance } \\
\qquad(12 \text {-week } \\
\text { cycles for } 2 \text { years } \\
\text { from IM I) }\end{array}$ & $\begin{array}{l}\text { Dexamethasone PO } \\
\text { Mercaptopurine PO } \\
\text { Methotrexate PO } \\
\text { Vincristine IV } \\
\text { Methotrexate IT }\end{array}$ & $\begin{array}{l}\text { Days I-5, 29-33, and 57-6I } \\
\text { Days I-84 } \\
\text { Weekly starting Day } 8 \\
\text { Days I, } 29,57 \\
\text { Day I }\end{array}$ \\
\hline
\end{tabular}

Abbreviations: PO, oral; IV, intravenous; IT, intrathecal; CNS, central nervous system.

reduced prevalence of genetic subtypes associated with favorable outcome and simultaneously an increase in subtypes associated with poor outcome. ${ }^{51}$ There is near-universal consensus from prior studies supporting the use of pegaspargase as the front-line asparaginase product in children and AYAs with newly diagnosed ALL (Tables 2 and 3).

\section{Relapsed ALL}

Apart from becoming an integral component of therapy regimens for first-line treatment of pediatric ALL, pegaspargase has also established a critical role in
Table 3 High-Risk B and T-ALL Regimens (Adapted from Children's Oncology Group AALLII3I, AALL043I)

\begin{tabular}{|c|c|c|}
\hline Phase & $\begin{array}{l}\text { Chemotherapy } \\
\text { Agents }\end{array}$ & Schedule \\
\hline $\begin{array}{l}\text { Induction } \\
\text { (35 days) }\end{array}$ & $\begin{array}{l}\text { Dexamethasone PO } \\
(<10 \text { years of age })^{\mathbf{a}} \\
\text { Prednisone PO }(\geq 10 \\
\text { years of age })^{\mathbf{a}} \\
\text { Vincristine IV } \\
\text { Daunorubicin IV } \\
\text { Pegaspargase IM/IV } \\
\text { Cytarabine IT } \\
\text { Methotrexate IT }\end{array}$ & $\begin{array}{l}\text { Days I-14 } \\
\text { Days I-28 } \\
\text { Days I, 8, I5, } 22 \\
\text { Days I, 8, I5, } 22 \\
\text { Day } 4 \\
\text { Day I (B-ALL CNS2 require } \\
\text { more) } \\
\text { Days 8, } 29 \text { (also Days I5, } 22 \\
\text { for CNS3) }\end{array}$ \\
\hline $\begin{array}{l}\text { Consolidation }^{\text {b }} \\
\text { ( } 56 \text { days for } \\
\text { B-ALL; } \\
77 \text { days for } \\
\text { T-ALL) }\end{array}$ & $\begin{array}{l}\text { Mercaptopurine PO } \\
\text { Cyclophosphamide IV } \\
\text { Cytarabine IV } \\
\text { Vincristine IV } \\
\text { Pegaspargase IM/IV } \\
\text { Methotrexate IT }\end{array}$ & $\begin{array}{l}\text { B-ALL: Days I-I4 and 29-42 } \\
\text { T-ALL: Days 8-2I and 50-63 } \\
\text { B-ALL: Days I, } 29 \\
\text { T-ALL: Days 8, } 50 \\
\text { B-ALL: Days I-4, 8-II, } \\
\text { 29-32, 36-39 } \\
\text { T-ALL: Days 8-II, I5-I8, } \\
\text { 50-53, 57-60 } \\
\text { B-ALL: Days I5, 22, 43, } 50 \\
\text { T-ALL: Days 22, 29, 64, 7I } \\
\text { B-ALL: Days I5, 43 } \\
\text { T-ALL: Days 22, 64 } \\
\text { B-ALL: Days I, 8, I5, 22 } \\
\text { T-ALL: Days I5, 22, 57, } 64 \\
\text { (CNS3 omit d22) }\end{array}$ \\
\hline $\begin{array}{l}\text { Interim } \\
\text { Maintenance } \\
\text { (IM) }^{\text {c }} \\
\quad(63 \text { days for } \\
\text { B-ALL; } \\
\quad 56 \text { days for } \\
\text { T-ALL) }\end{array}$ & $\begin{array}{l}\text { Mercaptopurine PO } \\
\text { Vincristine IV } \\
\text { High Dose } \\
\text { Methotrexate IV } \\
\text { Escalating Dose } \\
\text { Methotrexate IV } \\
\text { Methotrexate IT } \\
\text { Pegaspargase IV/IM }\end{array}$ & $\begin{array}{l}\text { B-ALL only: Days I-56 } \\
\text { B-ALL: Days I, I5, 29, } 43 \\
\text { T-ALL: Days I, II, 2I, 3I, 4I } \\
\text { B-ALL only: Days I, I5, 29, } 43 \\
\text { T-ALL only: Days I, II, 2I, } \\
\text { 3I, } 4 \text { I } \\
\text { B-ALL: Days I, } 29 \\
\text { T-ALL: Days I, 3I } \\
\text { T-ALL only: Days 2, } 22\end{array}$ \\
\hline $\begin{array}{l}\text { Delayed } \\
\text { Intensification } \\
\quad \text { ( } 56 \text { days for } \\
\text { B-ALL; } \\
\quad 63 \text { days for } \\
\text { T-ALL) }\end{array}$ & $\begin{array}{l}\text { Dexamethasone PO } \\
\text { Thioguanine PO } \\
\text { Vincristine IV } \\
\text { Doxorubicin IV } \\
\text { Pegaspargase IM/IV } \\
\text { Cyclophosphamide IV } \\
\text { Cytarabine IV } \\
\text { Methotrexate IT }\end{array}$ & $\begin{array}{l}\text { Days I-7 and I5-2I } \\
\text { B-ALL: Days 29-42 } \\
\text { T-ALL: Days 36-49 } \\
\text { Days I, 8, I5, 43, } 50 \text { (T-ALL } \\
\text { omit d43) } \\
\text { Days I, 8, I5 } \\
\text { B-ALL: Days 4, } 43 \\
\text { T-ALL: Days 4, } 50 \\
\text { Day } 29 \\
\text { Days } 29-32 \text { and 36-39 } \\
\text { Days I, 29, 36 (T-ALL d29 } \\
\text { given on d43) }\end{array}$ \\
\hline
\end{tabular}

(Continued) 
Table 3 (Continued).

\begin{tabular}{|c|c|c|}
\hline Phase & $\begin{array}{l}\text { Chemotherapy } \\
\text { Agents }\end{array}$ & Schedule \\
\hline $\begin{array}{l}\text { Maintenance }^{d} \\
\qquad(12 \text {-week } \\
\text { cycles for } 2 \text { years } \\
\text { from IM) }\end{array}$ & $\begin{array}{l}\text { Prednisone PO } \\
\text { Mercaptopurine PO } \\
\text { Methotrexate PO } \\
\text { Vincristine IV } \\
\text { Methotrexate IT }\end{array}$ & $\begin{array}{l}\text { Days I-5, 29-33, and 57-6I } \\
\text { Days I-84 } \\
\text { Weekly starting Day } 8 \\
\text { Days I, } 29,57 \\
\text { B-ALL: Days I, } 29 \text { (first } 4 \\
\text { cycles only) } \\
\text { T-ALL: Day I only }\end{array}$ \\
\hline
\end{tabular}

Notes: ${ }^{\mathrm{T} T}$-ALL patients receive prednisone only Days I-28. ${ }^{\mathrm{b}} \mathrm{T}$-ALL patients receive nelarabine Days I-5 and 43-47. ${ }^{\mathrm{C}} \mathrm{T}-\mathrm{ALL}$ patients receive nelarabine on Days 29-33. ${ }^{\mathrm{d}}$ T-ALL patients receive an additional 3 cycles of nelarabine-based therapy prior to the start of maintenance (each cycle 84 days).

Abbreviations: PO, oral; IM, intramuscular; IV, intravenous; IT, intrathecal; CNS, central nervous system.

treatment regimens for relapsed ALL. CHMC 1001 enrolled 28 pediatric patients with relapsed ALL at 3 pediatric institutions to study PK after pegaspargase therapy. ${ }^{52}$ Patients received induction therapy (including pegaspargase $2500 \mathrm{IU} / \mathrm{m}^{2} \mathrm{IM}$ weekly on days 2,9 , 16, and 23) and intensification therapy (including pegaspargase $2500 \mathrm{IU} / \mathrm{m}^{2} \mathrm{IM}$ once on day 7) (Table 1). Adequate serum and CSF asparagine depletion with SAA of $>0.1 \mathrm{IU} / \mathrm{mL}$ was observed and maintained during induction and intensification in the majority of samples. CCG 1941 utilized 2 doses of pegaspargase on days 2 and 16, in combination with multiagent chemotherapy during re-induction, to explore possible relationships among SAA, asparagine depletion, antiasparaginase antibody titers, and response to reinduction therapy in children and adolescents with early first bone marrow ALL relapse (Table 1). ${ }^{18}$ The study enrolled 214 patients between 1995-1998. The difference in asparagine levels between M1 and M3 response at end of re-induction was statistically significant $(\mathrm{p}=0.01)$. Lesser asparagine depletion was associated with failure to achieve second remissions. ${ }^{18}$ ALLR3 enrolled 216 patients aged 1-18 years in centers throughout Europe, Australia and New Zealand, on an open-label randomized trial for first ALL relapse, with a conventional four-drug induction and continuous asparagine depletion throughout the first 3 months. ALLR3 has since become the standard chemotherapy regimen for many relapsed ALL reinduction protocols. ${ }^{53}$
Table 4 Relapse ALL Regimen (Adapted from UKALLR3)

\begin{tabular}{|c|c|c|}
\hline Phase & $\begin{array}{l}\text { Chemotherapy } \\
\text { Agents }\end{array}$ & Schedule \\
\hline $\begin{array}{l}\text { Induction } \\
\qquad(28 \text { days) }\end{array}$ & $\begin{array}{l}\text { Dexamethasone PO } \\
\text { Vincristine IV } \\
\text { Mitoxantrone IV } \\
\text { Pegaspargase IM/IV } \\
\text { Methotrexate IT }\end{array}$ & $\begin{array}{l}\text { Days I-5, I5-19 } \\
\text { Days 3, 10, 17, } 24 \\
\text { Days I, } 2 \\
\text { Days 3, } 18 \\
\text { Days I, } 8 \text { (also Days } \\
\text { I5, } 22 \text { for CNS3) }\end{array}$ \\
\hline $\begin{array}{c}\text { Consolidation } \\
\text { (28 days) }\end{array}$ & $\begin{array}{l}\text { Dexamethasone PO } \\
\text { Vincristine IV } \\
\text { Methotrexate IV } \\
\text { Pegaspargase IM/IV } \\
\text { Cyclophosphamide IV } \\
\text { Etoposide IV } \\
\text { Methotrexate IT }\end{array}$ & $\begin{array}{l}\text { Days I-5 } \\
\text { Day } 3 \\
\text { Day } 8 \\
\text { Day } 9 \\
\text { Days 15-19 } \\
\text { Days 15-19 } \\
\text { Day } 8\end{array}$ \\
\hline $\begin{array}{l}\text { Intensification } \\
\text { (28 days) }\end{array}$ & $\begin{array}{l}\text { Dexamethasone PO } \\
\text { Vincristine IV } \\
\text { Cytarabine IV, every } \\
\text { I } 2 \text { hours } \\
\text { Erwinia ASP IM } \\
\text { Methotrexate IV } \\
\text { Methotrexate IT }\end{array}$ & $\begin{array}{l}\text { Days I-5 } \\
\text { Day } 3 \\
\text { Days I, 2, 8, } 9 \\
\text { Days 2, 4, 9, II, } 23 \\
\text { Day } 22 \\
\text { Days I, } 22\end{array}$ \\
\hline Before SCT & $\begin{array}{l}\text { Fludarabine IV } \\
\text { Cytarabine IV } \\
\text { Liposomal } \\
\text { Daunorubicin IV }\end{array}$ & $\begin{array}{l}\text { Days I-5 } \\
\text { Days I-5 } \\
\text { Day I }\end{array}$ \\
\hline $\begin{array}{l}\text { Before } \\
\text { Maintenance }^{\mathrm{a}} \\
\quad(\text { weeks 14-29) }\end{array}$ & $\begin{array}{l}\text { Dexamethasone PO } \\
\text { Vincristine IV } \\
\text { 6-mercaptopurine PO } \\
\text { Methotrexate PO } \\
\text { Etoposide IV } \\
\text { Cyclophosphamide IV } \\
\text { Cytarabine IV } \\
\text { Methotrexate IT }\end{array}$ & $\begin{array}{l}\text { Days I-5, 57-6I } \\
\text { Days 3, 59 } \\
\text { Days I-42, 57-98 } \\
\text { Days I0, I7, 3I, 38, } \\
67,74,88,95 \\
\text { Days 42, 49, 99, } 106 \\
\text { Days 42, 49, 99, } 106 \\
\text { Days 43-46, 50-53, } \\
\text { 100-103, 107-1 } 10 \\
\text { Days I, 43, 57, } 99\end{array}$ \\
\hline $\begin{array}{l}\text { Maintenance }^{\mathrm{a}} \\
\quad(4-\text { week cycles } \\
\text { from weeks } \\
\text { 30-104) }\end{array}$ & $\begin{array}{l}\text { Dexamethasone PO } \\
\text { Mercaptopurine PO } \\
\text { Methotrexate PO } \\
\text { Vincristine IV } \\
\text { Methotrexate IT }\end{array}$ & $\begin{array}{l}\text { Days I-5 } \\
\text { Days I-28 } \\
\text { Weekly } \\
\text { Day I } \\
\text { Day I }\end{array}$ \\
\hline
\end{tabular}

Note: aPhase given to patients who did not go to SCT.

Abbreviations: PO, oral; IV, intravenous; IM, intramuscular; IT, intrathecal; CNS, central nervous system; SCT, stem cell transplant; ASP, asparaginase.

Pegaspargase is an important chemotherapy for treating pediatric ALL relapse and is included in the majority of US and European re-induction protocols with the ALLR3 or vincristine, dexamethasone, pegaspargase and doxorubicin (VXLD) inspired backbones (Tables 4 and 5). 
Table 5 Relapse ALL Regimen* (Adapted from VXLD ReInduction)

\begin{tabular}{|c|c|c|}
\hline Phase & $\begin{array}{l}\text { Chemotherapy } \\
\text { Agents }\end{array}$ & Schedule \\
\hline $\begin{array}{l}\text { Induction } \\
\text { (28 days) }\end{array}$ & $\begin{array}{l}\text { Dexamethasone PO } \\
\text { Vincristine IV } \\
\text { Doxorubicin IV } \\
\text { Pegaspargase IM/IV } \\
\text { Methotrexate IT } \\
\text { Cytarabine IT }\end{array}$ & $\begin{array}{l}\text { Days I-14 } \\
\text { Days I, 8, 15, } 22 \\
\text { Day I } \\
\text { Days 2, } 16 \\
\text { Day I } \\
\text { Day I }\end{array}$ \\
\hline $\begin{array}{c}\text { Consolidation }^{\mathrm{a}} \\
\text { (28 days) }\end{array}$ & $\begin{array}{l}\text { Dexamethasone PO } \\
\text { Vincristine IV } \\
\text { Methotrexate IV } \\
\text { Pegaspargase IM/IV } \\
\text { Cyclophosphamide IV } \\
\text { Etoposide IV } \\
\text { Methotrexate IT }\end{array}$ & $\begin{array}{l}\text { Days I-5 } \\
\text { Day } 3 \\
\text { Day } 8 \\
\text { Day } 9 \\
\text { Days 15-19 } \\
\text { Days 15-19 } \\
\text { Day } 8\end{array}$ \\
\hline $\begin{array}{c}\text { Consolidation }{ }^{\mathrm{b}} \\
\text { (56 days) }\end{array}$ & $\begin{array}{l}\text { Mercaptopurine PO } \\
\text { Cyclophosphamide IV } \\
\text { Cytarabine IV } \\
\text { Vincristine IV } \\
\text { Pegaspargase IM/IV } \\
\text { Methotrexate IT }\end{array}$ & $\begin{array}{l}\text { Days I-14 and 29-42 } \\
\text { Days I, } 29 \\
\text { Days I-4, 8-II, 29-32, } \\
\text { and } 36-39 \\
\text { Days I5, 22, 43, } 50 \\
\text { Days I5, 43 } \\
\text { Days I, 8, I5, } 22\end{array}$ \\
\hline
\end{tabular}

Notes: *Regimen used as backbone for relapsed ALL after window with novel agents eg, Bortezomib, Carfilzomib, Pevonedistat, Daratumumab, Blinatumomab, and Nivolumab. ${ }^{\mathrm{a}, \mathrm{b}}$ Depending on study protocol. ${ }^{\mathrm{b}}$ Consolidation based on modified BFM.

Abbreviations: VXLD, vincristine, dexamethasone, pegaspargase, doxorubicin; PO, oral; IV, intravenous; IM, intramuscular; IT, intrathecal.

\section{Toxicity of Pegaspargase}

In addition to its multiple clinical benefits, pegaspargase is notable for significant toxicity in up to $20-25 \%$ of all patients. $^{54,55}$ These toxicities include hypersensitivity, pancreatitis, hyperglycemia, hyperlipidemia, liver dysfunction, hyperbilirubinemia, thrombosis, bleeding, and osteonecrosis (Figure 1).

\section{Hypersensitivity to Pegaspargase}

Allergic and infusion-related reactions in patients receiving asparaginase therapy are among the most common toxicities, ranging between $3-71 \%$ (Figure 1). ${ }^{56}$ Asparaginase hypersensitivity is mediated by antigen-specific $\operatorname{IgG}$ and/or $\mathrm{IgE}$ through the immunoglobulin receptors FcyRIII and FceRI, respectively. ${ }^{57}$ Hypersensitivity reactions to $E$. coli derived asparaginase, necessitate a switch to an alternative preparation such as Erwinia asparaginase. ${ }^{8,56,58}$ The incidence of hypersensitivity reactions depends on the number of prior exposures, interval between doses, type of asparaginase, use of premedication, and concomitant corticosteroid therapy. There are two types of true hypersensitivity reactions, antibody production with clinical manifestations and neutralizing antibody production reflected by a day 7 SAA below 0.1 $\mathrm{IU} / \mathrm{mL}$ and/or a day 14 level below the lower limit of quantification with an absence of a clinical reaction, referred to as silent inactivation or subclinical hypersensitivity. ${ }^{32}$ The antibody formed to the naïve asparaginase or polyethylene glycol portion of the pegaspargase alters the PK of the drug. ${ }^{59,60}$ The presence of these antibodies can inhibit asparaginase from depleting asparagine and ultimately decrease efficacy. Several studies suggest that pegaspargase is less immunogenic than the native $E$. coli formulation. ${ }^{3,60-62}$

The above referenced DFCI 91-01 study reported allergic reaction in $15 \%$ of patients; however, pegaspargase was associated with a lower incidence of allergic reactions $(p=0.02){ }^{3}$

The Nordic Society of Paediatric Haematology and Oncology (NOPHO) ALL2008 protocol enrolled 106 pediatric patients from 2008-2012. Six patients experienced a clinical allergic reaction to pegaspargase, a cumulative risk of $13.2 \%$. Reactions usually occurred 2 hours after IM administration with symptoms ranging from mild to severe anaphylaxis. ${ }^{63}$ Additionally, NOPHO ALL2008, through a genome-wide association study (GWAS), correlated several genetic variants to an increased incidence of pegaspargase hypersensitivity. ${ }^{64}$

The Dutch Childhood Oncology Group (DCOG) ALL10 study enrolled children ages 1-18 years with newly diagnosed ALL from 2009-2012. ${ }^{65}$ Patients were stratified into 3 risk groups after induction therapy (standard, medium and high) and all received 8 doses of native $E$. coli asparaginase ( $5000 \mathrm{IU} / \mathrm{m}^{2}$ per dose) every 3 days in induction. Eighty-nine patients considered medium risk were given pegaspargase as first-line agent $\left(2500 \mathrm{IU} / \mathrm{m}^{2}\right.$ per dose every other week) for a total of 15 doses during first 30 weeks of intensification. In the setting of allergy or silent inactivation, pegaspargase was replaced with Erwinia asparaginase. The investigators found a high incidence of inactivation of pegaspargase (22\% clinical allergy and $8 \%$ silent inactivation) in the intensification phase due to antibody development against native $E$. coli asparaginase used in induction. They concluded that if a second pegaspargase course was administered, the allergy rate of pegaspargase would increase. ${ }^{65}$ DCOG ALL-11 study reported 14 allergic-like reactions or infusion reactions, 5 were to pegaspargase and 9 to Erwinia asparaginase. Allergic-like reactions occurred relatively late after the 


\begin{tabular}{|c|c|}
\hline Toxicity & Studies \\
\hline Hypersensitivity & $\begin{array}{l}\text { DFCI } 91-01^{3} \\
\text { NOPHO ALL2008 } \\
\text { DCOG ALL-10 }\end{array}$ \\
\hline Pancreatitis & $\begin{array}{l}\text { DFCl 87-01, 91-01, 95-01, 00-01 } 79 \\
\text { UKALL } 2003^{80} \\
\text { DCOG ALL-10 } \\
\text { NOPHO ALL2008 } \\
\text { }{ }^{81}\end{array}$ \\
\hline Hyperglycemia & POG $9203^{86 *}$ \\
\hline Hyperlipidemia & $\begin{array}{l}\text { Total XV, XVI }{ }^{90} \\
\text { DCOG ALL-10 }\end{array}$ \\
\hline Hepatotoxicity & $\begin{array}{l}\text { NOPHO ALL2008 } 95 \\
\text { DCOG ALL-11 } 96\end{array}$ \\
\hline $\begin{array}{l}\text { Thrombosis/ } \\
\text { Bleeding }\end{array}$ & $\begin{array}{l}\text { DFCI 91-01, 95-01, 00-01, 05-01101 } \\
\text { CCG } 1962^{32} \\
\text { DCOG ALL-10 } 102\end{array}$ \\
\hline Osteonecrosis & $\begin{array}{l}\text { Total XV } 107 \\
\text { NOPHO ALL2008 } 108,109 \\
\text { COG AALL0331 } 110\end{array}$ \\
\hline
\end{tabular}

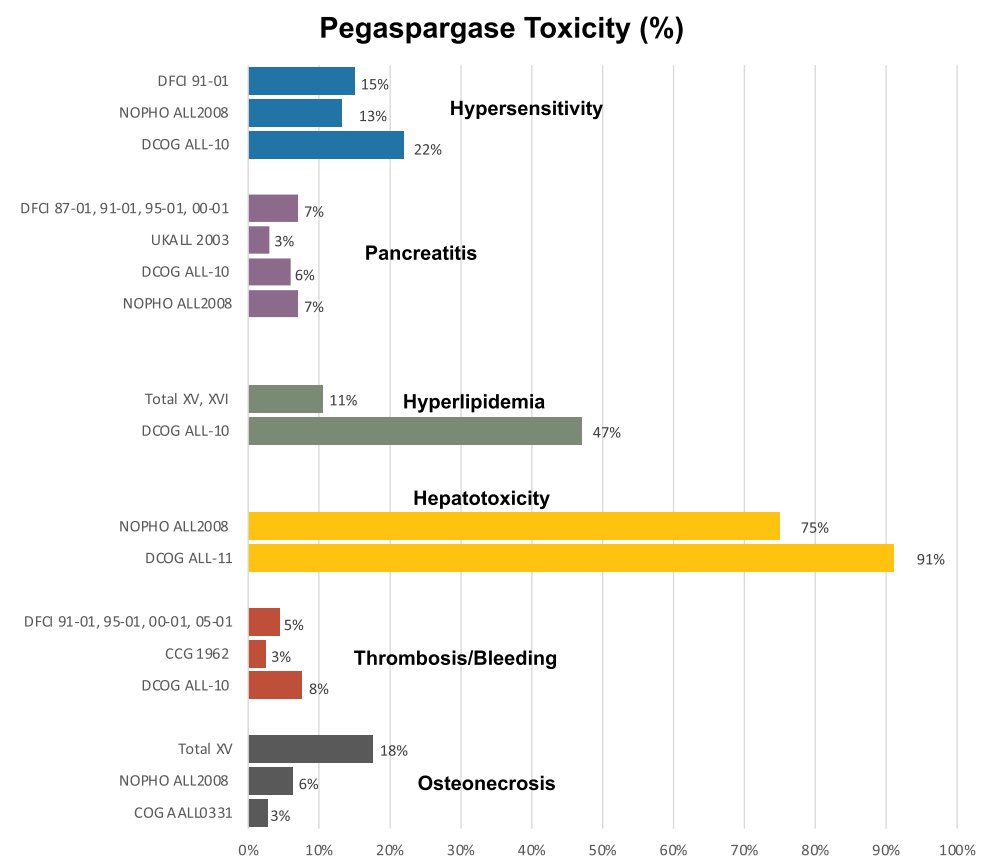

Figure I Pegaspargase toxicity. *Due to lack of reported percentages for specific toxicities, hyperglycemia is not included.

start of infusion when compared to real allergies. They also were distinguished from real allergies by the absence of antibodies in all but one patient with an allergic-like reaction, while antibodies were detected in all patients with a real allergy. The study recommended if clinically tolerated, formulations should not be switched in case of allergic-like reactions. ${ }^{66}$

POG 8866 enrolled 76 patients $<21$ years with ALL in second bone marrow relapse from 1988-1992, with the aim of comparing efficacy and toxicity of pegaspargase to native $E$. coli asparaginase in the standard re-induction regimen. ${ }^{67}$ It was noted that all patients hypersensitive to native asparaginase tolerated at least 2 doses of pegaspargase given during induction phase. In those with known hypersensitivity to native asparaginase, increased clearance and decreased efficacy of pegaspargase were observed. ${ }^{67}$ Recently multiple studies have shown monitoring of ASNase activity $\geq 0.1 \mathrm{IU} / \mathrm{mL}$, measured on days 7 and 14 post pegaspargase administration, during treatment of children with ALL is feasible and informative, demonstrating median trough PEG-ASNase activity being high in all patients without hypersensitivity. ${ }^{32,68,69}$

COG AALL1421 results suggest pre-existing anti-PEG antibodies with the increased use of pegylated products in food processing and cosmetics may also play a role in hypersensitivity reactions. ${ }^{40,41,70}$
Pegaspargase is a critical component of the chemotherapeutic backbone in upfront ALL protocols (Tables 2 and 3 ), and those patients who experience hypersensitivity to pegaspargase should discontinue any further dosing and switch to Erwinia asparaginase. ${ }^{71}$ However, during an Erwinia asparaginase shortage, Verma et al conducted a successful prospective study of pegaspargase desensitization in 10 patients with ALL/LLy who had experienced prior grade 3 or higher hypersensitivity reactions to either pegaspargase (6) or both pegaspargase and Erwinia asparaginase (4). A 13-step rapid desensitization protocol was utilized and SAA levels were measured between days 4-7 and days $10-14 .{ }^{72}$ Seven patients had sustained SAA activity, one achieved therapeutic levels but showed accelerated clearance, while two did not achieve adequate levels consistent with neutralizing antibodies. Although three of the 10 patients had mild to moderate hypersensitivity reactions, all were able to receive full protocol doses after clinical intervention. Others have since duplicated the safe administration of pegaspargase, while maintaining sustained SAA levels, utilizing a desensitization protocol in patients experiencing prior hypersensitivity reactions. ${ }^{72-74}$

An additional area of investigation for patients who experience hypersensitivity is the COG protocol AALL1931 (NCT04145531) an open-label, multicenter study for patients with ALL/LLy following hypersensitivity 
to $E$. coli derived asparaginase with the objective to determine the efficacy, safety, and tolerability of IM recombinant crisantaspase Pseudomonas fluorescens.

\section{Pancreatitis}

Asparaginase-associated pancreatitis (AAP) was first reported in patients receiving the native L-asparaginase formulation. The origin, formulation, dosage and method of administration do not seem to influence the risk of pancreatitis. $^{75}$ The exact pathophysiology of AAP is unknown, but it is believed to be caused by a systemic depletion of asparagine and thus reduction of protein synthesis. Organs with increased protein turnover such as the pancreas and liver are most at risk. Incidence of AAP is between $7-18 \%$ with it being more frequent in the AYA ALL population (Figure 1) and is one of the most common causes of truncation of asparaginase therapy, mostly due to the high chances of recurrence of AAP after rechallenge. ${ }^{76-78}$

Kearney et al reviewed the clinical course of all children with ALL diagnosed with pancreatitis at DFCI from $1987-2003 .{ }^{79}$ A total of 1583 patients aged $0-18$ years were enrolled in DFCI ALL consortium protocols (87-01, 91-01, 95-01, 00-01). Twenty-eight (7\%) out of 403 patients treated at Boston Children's Hospital and $74(6.4 \%)$ of 1155 patients in 10 other centers were diagnosed with at least one episode of AAP. Those patients with AAP had a higher median age, indicating patients $10-18$ years of age have 2.4 times increased risk of developing pancreatitis. There was a non-statistically significant trend towards inferior 5 -year EFS, with $29 \%$ of patients with history of AAP subsequently relapsing compared to only $14 \%$ without AAP. ${ }^{79}$ UKALL 2003 enrolled 3101 patients aged 1-25 years on 3 risk-stratified arms. ${ }^{80} \mathrm{SR}$ and IR patients were exposed to a relatively small amount of pegaspargase $\left(3000-4000 \mathrm{IU} / \mathrm{m}^{2}\right)$ and $\mathrm{HR}$ patients received $12,000 \mathrm{IU} / \mathrm{m}^{2}$. Pancreatitis was more common in asparaginase-containing blocks versus non-asparaginase containing blocks ( $83 \%$ vs. $17 \%$; p $<0.0001)$. The median interval between receiving pegaspargase dose and developing AAP was 10 days. There was significant morbidity in the setting of AAP evidenced by increased readmission rates $(29 \%)$, pseudocysts $(25 \%)$, diabetes mellitus (13\%), pleural effusions (8\%), and renal failure $(2 \%)$. Further pegaspargase was omitted in patients with higher grade AAP. The risk for pancreatitis was associated with increasing intensity of asparaginase treatment and dose, as well as increasing age. ${ }^{80}$ Similar results were observed by the NOPHO ALL2008 study with incidences of AAP higher in AYAs (10.1\%) compared to in children $(7 \%, \mathrm{p}=0.03)$ and $44 \%$ developed recurrence of AAP on rechallenge with asparaginase. ${ }^{81}$ An observational Ponte di Legno Toxicity Working Group reviewed patients on 26 trials conducted by 18 trial groups with children (1-18 years) between 1996-2016, to investigate the risk of complications and risk of re-exposing patients with $\mathrm{AAP}^{82}$ Complications noted in the 465 patients with AAP included mechanical ventilation (8\%), pseudocysts (26\%), acute insulin need $(21 \%)$, and death $(2 \%)$. Older age was associated with more complications (10.5 years vs. 6.1 years without complications; $p<0.0001)$. One year after diagnosis of AAP, $11 \%$ of patients continued to need insulin, had recurrent abdominal pain, or both. Ninety-six patients were re-exposed to asparaginase, including 59 after severe AAP, and 44 (46\%) patients developed a second episode, 22 (52\%) being severe, suggesting a high risk of recurrence. ${ }^{82}$

Patients with Common Terminology Criteria for Adverse Events (CTCAE) grade 1/2 AAP can be rechallenged after resolution, whereas it is recommended to omit further pegaspargase therapy in severe grades 3/4 AAP, as $44-63 \%$ have risk of recurrence on reexposure. $^{75,79,81,82}$

\section{Hyperglycemia}

Pegaspargase-induced hyperglycemia is believed to be due to decreased insulin production and modification or deficiency of insulin receptors. ${ }^{83}$ The use of concomitant steroids and pegaspargase is believed to cause potentiation of hepatic gluconeogenesis and insulin resistance, thereby worsening hyperglycemia. ${ }^{84,85}$

POG 9203 enrolled 34 patients between the ages of 1-21 years from 1992-1993. This study's aims included feasibility determination of 29 biweekly doses of pegaspargase on a backbone of intense multiagent antimetabolite consolidation and maintenance in HR B-ALL. Excessive toxicities attributed to pegaspargase and myelosuppression were encountered during consolidation, causing the early closure of this study. Six patients developed hyperglycemia. The greatest risk factor appeared to be concomitant use of steroid therapy (Figure 1) ${ }^{86}$

Although insulin therapy may be required in severe cases, severe hyperglycemia is usually transient and does not require truncation of pegaspargase therapy. ${ }^{87}$ 


\section{Hyperlipidemia}

Asparaginase and steroids during ALL therapy lead to abnormalities in lipid metabolism, most notably resulting in hypertriglyceridemia and/or hypercholesterolemia. ${ }^{88}$ The exact mechanism for lipid dysregulation with asparaginase therapy is not known, but it is postulated to be due to an increase in the endogenous hepatic synthesis of very low-density lipoproteins. Another reason may be decreased activity in lipoprotein lipase, an enzyme involved in the removal of triglyceride-rich lipoproteins from the plasma. ${ }^{89}$ St. Jude Children's Research Hospital evaluated different asparaginase formulations in patients $\leq 18$ years of age with ALL on two trials; Total XV conducted from 2000-2007 and Total XVI from 2007-2017, enrolling 498 and 598 patients, respectively. SR/HR patients treated with pegaspargase had more severe hypertriglyceridemia compared to native L-asparaginase, and the highest increase occurred when pegaspargase was given in combination with dexamethasone. ${ }^{90}$ DCOG ALL 10 reported $47 \%$ of patients receiving pegaspargase developed hypertriglyceridemia compared to $0 \%$ receiving Erwinia asparaginase, thereby suggesting prolonged exposure to asparaginase therapy was associated with hypertriglyceridemia (Figure 1). ${ }^{91}$

Most patients have transient, asymptomatic hypertriglyceridemia. Omission or alteration of pegaspargase therapy is not recommended, though hypertriglyceridemia greater than $2000 \mathrm{mg} / \mathrm{dL}$ can enhance risk of AAP due to severe chylomicronemia. ${ }^{89}$ Preventive measures like dietary restrictions, fibrates, insulin infusions, heparin infusions, and plasmapheresis have been attempted, but their success in preventing AAP or other morbidities is not established. ${ }^{92,93}$

\section{Hepatotoxicity}

Hepatic toxicities encountered with pegaspargase include direct damage to hepatocytes (elevated hepatic transferases), cholestatic injury (elevated alkaline phosphatase and bilirubin), and impaired synthetic function (decreases in antithrombin, fibrinogen, and albumin with increases in cholesterol, phospholipids and triglycerides). The hepatotoxicity caused by asparaginase is believed to be due to abnormal mitochondrial function in the liver, alterations in lipoprotein metabolism, and secretion. ${ }^{94}$

Though $75 \%$ patients had grade $1 / 2$ elevation in bilirubin, sinusoidal obstructive syndrome (SOS) was noted in $27 \%$, during NOPHO ALL2008 in patients undergoing maintenance treatment for ALL after the introduction of extended pegaspargase treatment. These findings prompted the hypothesis that pegaspargase in combination with other medications, such as mercaptopurine used during maintenance, might trigger SOS. ${ }^{95}$ DCOG ALL-11 study noted more patients developed grade 1-2 increases in ALT and AST (91\%) during asparaginase with high dose methotrexate courses, but not grade 3-4 hepatotoxicity (9\%) (Figure 1). ${ }^{96}$

Most protocols do not recommend alteration in pegaspargase therapy due to hepatotoxicity, although this is another reason to consider dose capping in AYA patients. There are case reports of improvement in hepatic function with use of levocarnitine, a mitochondrial co-factor and vitamin B therapy. ${ }^{97}$

\section{Thrombosis and Bleeding}

Asparaginase has simultaneous effects on procoagulant and thrombolytic proteins causing an increase in both thrombosis and bleeding. Asparaginase-induced depletion of asparagine in the serum leads to decreased synthesis of fibrinogen, plasminogen, anti-coagulation factor antithrombin III (ATIII), protein $\mathrm{C}$, and protein $\mathrm{S}$ leading to increased thrombin formation. ${ }^{98}$ Additional factors contributing to the risk include concomitant steroid therapy particularly prednisone, indwelling central venous catheter, genetic thrombophilia, and underlying ALL itself. ${ }^{99,100}$

DFCI studied 501 patients between 1991-2008 with newly diagnosed ALL. These patients were enrolled in four pediatric ALL protocols (91-01, 95-01, 00-01, and 05-01) and adult protocol $01-175 .{ }^{101}$ Five percent of pediatric patients and $34 \%$ of adult patients experienced thrombosis during treatment (Figure 1). The risk of thrombosis was noted to increase with age, with further risk stratification indicating a high risk for those $>10$ years of age and extremely high risk for those $>30$ years of age. CCG 1962 studied efficacy and PK of a single IM dose of pegaspargase instead of multiple IM doses of native $E$. coli asparaginase in 3 phases of SR ALL therapy. An incidence of cerebral venous sinus thrombosis (CVST) of $2-3 \%$ was observed for both regimens. ${ }^{24}$ DCOG ALL-10 enrolled 778 ALL patients between 2004-2013 and demonstrated VTE in $7.6 \%$ of patients with CVST in $44.1 \%$ of these VTE patients. ${ }^{102}$ Age $>7$ years, T-ALL, steroids, and length of exposure to pegaspargase were the main risk factors. The presence of VTE did not impact EFS or OS. ${ }^{101,102}$

Patients with non-catheter-associated VTE should have asparaginase therapy withheld until imaging shows VTE and 
CVST resolution. Patients should be treated with anticoagulation, preferably low-molecular-weight heparin, and upon resolution or stabilization of thrombus, consideration may be given to rechallenging with pegaspargase. ${ }^{100,101}$

\section{Osteonecrosis}

Osteonecrosis is a known complication in 15-45\% of patients, especially AYA patients, undergoing treatment for ALL. ${ }^{103,104}$ Glucocorticoids, notably dexamethasone, are believed to induce inhibition of angiogenesis, bone marrow adipogenesis, hypercoagulation, and apoptosis of endothelial cells and osteocytes. ${ }^{105}$ Asparaginase decreases the clearance of dexamethasone due to its hypoproteinemia effect. Additionally, the immunosuppressive effects of glucocorticoids inhibit the antibody response against asparaginase and prevent a neutralizing effect causing higher SAA. ${ }^{105}$ The potentiated effect of asparaginase on glucocorticoidinduced osteonecrosis is believed to be in the setting of a hypercoagulable state which can lead to impaired circulation, vascular damage, and subsequent osteonecrosis. ${ }^{106}$

Liu et al were the first to study the effects of asparaginase treatment on dexamethasone-induced osteonecrosis utilizing a mouse model. ${ }^{105}$ St. Jude's Total XV protocol noted that patients with antibodies against asparaginase had a lower risk of developing osteonecrosis than those without the antibodies. ${ }^{107}$

Osteonecrosis 5-year cumulative incidence (CI) was $6.3 \%$ on NOPHO ALL2008 and the patients in the experimental arm who did not receive pegaspargase during dexamethasone containing DI had reduced frequency of osteonecrosis (Figure 1). ${ }^{108}$ NOPHO ALL2008 also correlated hyperlipidemia with osteonecrosis. ${ }^{109}$ Prolonged hyperlipidemia was associated with a significantly higher osteonecrosis specific hazard ratio (HR) per $\mathrm{mmol} / \mathrm{L}$ for hypertriglyceridemia $(\mathrm{HR}=1.08, \mathrm{p}=0.038)$ as well as for cholesterol levels $(\mathrm{HR}=1.26, \mathrm{p}=0.039)$, and a tendency towards an inverse association with higher $\mathrm{HDL} \quad(\mathrm{HR}=0.22$, $\mathrm{p}=0.053) .{ }^{109}$ The COG AALL0331 SR trial enrolled 5261 patients between 2005-2010. ${ }^{110}$ Osteonecrosis 5-year overall CI was $2.7 \%$. Extended exposure to pegaspargase was a likely contributing factor, possibly by potentiating dexamethasone exposure during DI. The study concluded the risk for osteonecrosis can be significantly reduced by using alternate week dexamethasone during DI, which is now standard on all COG ALL protocols. ${ }^{110}$

Further studies are needed to elucidate whether lowering triglycerides and cholesterol during combined pegaspargase and dexamethasone therapy can reduce the risk of osteonecrosis.

\section{Conclusion}

Pegaspargase is an essential chemotherapeutic agent in all newly diagnosed pediatric and AYA ALL protocols, as well as most re-induction protocols for relapsed ALL. It is imperative to be aware of its toxicities and their management, so as to improve survival and decrease long-term morbidities in pediatric and AYA ALL. Areas of focus for future research should include asparaginase PK/PD of newer preparations, dosing regimens, interventions to mitigate toxicity, and role of genetics as it relates to asparaginase dosing and toxicity.

\section{Disclosure}

Anupam Verma, Cynthia Bender and Maria Carter have no conflicts of interest to report in this work. Luke Maese has served as consultant and has received honoraria from Jazz Pharmaceuticals.

\section{References}

1. Pui CH, Robison LL, Look AT. Acute lymphoblastic leukaemia. Lancet. 2008;371(9617):1030-1043. doi:10.1016/S0140-6736(08)60 457-2

2. Silverman LB, Stevenson KE, O'Brien JE, et al. Long-term results of Dana-Farber cancer institute ALL consortium protocols for children with newly diagnosed acute lymphoblastic leukemia (1985-2000). Leukemia. 2010;24(2):320-334. doi:10.1038/leu.20 09.253

3. Silverman LB, Gelber RD, Dalton VK, et al. Improved outcome for children with acute lymphoblastic leukemia: results of Dana-Farber consortium protocol 91-01. Blood. 2001;97(5): 1211-1218. doi:10.1182/blood.v97.5.1211

4. Pui CH, Sandlund JT, Pei D, et al. Improved outcome for children with acute lymphoblastic leukemia: results of total therapy study XIIIB at St jude children's research hospital. Blood. 2004;104(9):2690-2696. doi:10.1182/blood-2004-04-1616

5. Brown P, Inaba H, Annesley C, et al. Pediatric acute lymphoblastic leukemia, version 2.2020, NCCN clinical practice guidelines in Oncology. $J$ Natl Compr Canc Netw. 2020;18(1):81-112. doi:10.6004/jncen.2020.0001

6. Mattano LA, Devidas M, Friedmann AM, et al. Outstanding outcome for children with Standard Risk-Low (SR-Low) Acute Lymphoblastic Leukemia (ALL) and No Benefit to Intensified Peg-Asparaginase (PEG-ASNase) Therapy: results of Children's Oncology Group (COG) Study AALL0331. Blood. 2014;124(21):793. doi:10.1182/ blood.V124.21.793.793

7. Sallan SE, Hitchcock-Bryan S, Gelber R, Cassady JR, Frei E 3rd, Nathan DG. Influence of intensive asparaginase in the treatment of childhood non-T-cell acute lymphoblastic leukemia. Cancer Res. 1983;43(11):5601-5607.

8. Egler RA, Ahuja SP, Matloub Y. L-asparaginase in the treatment of patients with acute lymphoblastic leukemia. $J$ Pharmacol Pharmacother. 2016;7(2):62-71. doi:10.4103/0976-500X.184769

9. Kawedia JD, Rytting ME. Asparaginase in acute lymphoblastic leukemia. Clin Lymphoma Myeloma Leuk. 2014;14(Suppl):S14-S17. doi:10.1016/j.clml.2014.06.017 
10. Pui $\mathrm{CH}$, Campana D, Pei D, et al. Treating childhood acute lymphoblastic leukemia without cranial irradiation. $N$ Engl J Med. 2009;360(26):2730-2741. doi:10.1056/NEJMoa0900386

11. Pieters R, Carroll WL. Biology and treatment of acute lymphoblastic leukemia. Hematol Oncol Clin North Am. 2010;24 (1):1-18. doi:10.1016/j.hoc.2009.11.014

12. Clavell LA, Gelber RD, Cohen HJ, et al. Four-agent induction and intensive asparaginase therapy for treatment of childhood acute lymphoblastic leukemia. $N$ Engl $J$ Med. 1986;315 (11):657-663. doi:10.1056/NEJM198609113151101

13. Amylon MD, Shuster J, Pullen J, et al. Intensive high-dose asparaginase consolidation improves survival for pediatric patients with $\mathrm{T}$ cell acute lymphoblastic leukemia and advanced stage lymphoblastic lymphoma: a Pediatric Oncology Group study. Leukemia. 1999;13(3):335-342. doi:10.1038/sj.leu.2401310

14. Duval M, Suciu S, Ferster A, et al. Comparison of Escherichia coli-asparaginase with Erwinia-asparaginase in the treatment of childhood lymphoid malignancies: results of a randomized European Organisation for Research and Treatment of CancerChildren's Leukemia Group Phase 3 trial. Blood. 2002;99 (8):2734-2739. doi:10.1182/blood.v99.8.2734

15. Pession A, Valsecchi MG, Masera G, et al. Long-term results of a randomized trial on extended use of high dose L-asparaginase for standard risk childhood acute lymphoblastic leukemia. J Clin Oncol. 2005;23(28):7161-7167. doi:10.1200/ jco.2005.11.411

16. Hefazi M, Litzow MR. Recent advances in the biology and treatment of T cell acute lymphoblastic leukemia. Curr Hematol Malig Rep. 2018;13(4):265-274. doi:10.1007/s11899-018-0455-9

17. Schrappe M, Möricke A, Reiter A, et al. Key treatment questions in childhood acute lymphoblastic leukemia: results in 5 consecutive trials performed by the ALL-BFM study group from 1981 to 2000. Klin Padiatr. 2013;225(Suppl S 01):S62-S72. doi:10.1055/ s-0033-1337966

18. Jarrar M, Gaynon PS, Periclou AP, et al. Asparagine depletion after pegylated E. coli asparaginase treatment and induction outcome in children with acute lymphoblastic leukemia in first bone marrow relapse: a Children's Oncology Group study (CCG-1941). Pediatr Blood Cancer. 2006;47(2):141-146. doi:10.1002/pbc.20713

19. Avramis VI, Tiwari PN. Asparaginase (native ASNase or pegylated ASNase) in the treatment of acute lymphoblastic leukemia. Int J Nanomedicine. 2006;1(3):241-254.

20. Gupta S, Wang C, Raetz EA, et al. Impact of asparaginase discontinuation on outcome in childhood acute lymphoblastic leukemia: a report from the children's Oncology Group. J Clin Oncol. 2020;38(17):1897-1905. doi:10.1200/JCO.19.03024

21. European Medicines Agency. Oncaspar (pegaspargase): summary of product characteristics. 2018. Available from: http://www.ema. europa.eu/. Accessed March 15, 2021.

22. Shire Pharmaceuticals. Oncaspar (pegaspargase): US prescribing information. 2019. Available from: http://www.fda.gov. Accessed March 15, 2021.

23. Dinndorf PA, Gootenberg J, Cohen MH, Keegan P, Pazdur R. FDA drug approval summary: pegaspargase (oncaspar) for the first-line treatment of children with acute lymphoblastic leukemia (ALL). Oncologist. 2007;12(8):991-998. doi:10.1634/theoncologist.12-8991

24. Avramis V, Sencer S, Periclou A, et al. A randomized comparison of native Escherichia coli asparaginase and polyethylene glycol conjugated asparaginase for treatment of children with newly diagnosed standard-risk acute lymphoblastic leukemia: a Children's Cancer Group study. Blood. 2002;99:1986-1994. doi:10.1182/blood.V99.6.1986

25. Heo YA, Syed YY, Keam SJ. Pegaspargase: a review in acute lymphoblastic leukaemia. Drugs. 2019;79(7):767-777. doi:10.1007/s40265-019-01120-1
26. Asselin BL. The three asparaginases. Comparative pharmacology and optimal use in childhood leukemia. Adv Exp Med Biol. 1999;457:621-629.

27. Broome JD. Evidence that the L-asparaginase of guinea pig serum is responsible for its antilymphoma effects. I. Properties of the L-asparaginase of guinea pig serum in relation to those of the antilymphoma substance. $J$ Exp Med. 1963;118(1):99-120. doi:10.1084/jem.118.1.99

28. Schwartz JH, Reeves JY, Broome JD. Two L-asparaginases from E. coli and their action against tumors. Proc Natl Acad Sci U S A. 1966;56(5):1516-1519. doi:10.1073/pnas.56.5.1516

29. Boyse EA, Old LJ, Campbell HA, Mashburn LT. Suppression of murine leukemias by L-asparaginase. Incidence of sensitivity among leukemias of various types: comparative inhibitory activities of guinea pig serum L-asparaginase and Escherichia coli L-asparaginase. J Exp Med. 1967;125(1):17-31. doi:10.1084/ jem.125.1.17

30. Meneguetti GP, Santos JHPM, Obreque KMT, et al. Novel site-specific PEGylated L-asparaginase. PLoS One. 2019;14(2): e0211951. doi:10.1371/journal.pone.0211951

31. Lebovic R, Pearce N, Lacey L, Xenakis J, Faircloth CB, Thompson P. Adverse effects of pegaspargase in pediatric patients receiving doses greater than 3750 IU. Pediatr Blood Cancer. 2017;64(10):e26555. doi:10.1002/pbc.26555

32. van der Sluis IM, Vrooman LM, Pieters R, et al. Consensus expert recommendations for identification and management of asparaginase hypersensitivity and silent inactivation. Haematologica. 2016;101(3):279-285. doi:10.3324/ haematol.2015.137380

33. Place AE, Stevenson KE, Vrooman LM, et al. Intravenous pegylated asparaginase versus intramuscular native Escherichia coli L-asparaginase in newly diagnosed childhood acute lymphoblastic leukaemia (DFCI 05-001): a randomised, open-label phase 3 trial. Lancet Oncol. 2015;16(16):1677-1690. doi:10.1016/S14702045(15)00363-0

34. Schore RJ, Devidas M, Bleyer A, et al. Plasma asparaginase activity and asparagine depletion in acute lymphoblastic leukemia patients treated with pegaspargase on Children's Oncology Group AALL07P4. Leuk Lymphoma. 2019;60(7):1740-1748. doi:10.1080/10428194.2018.1542146

35. Vrooman LM, Blonquist TM, Supko JG, et al. Efficacy and toxicity of pegaspargase and calaspargase pegol in childhood acute lymphoblastic leukemia/lymphoma: results of DFCI 11-001. J Clin Oncol. 2019;37(15_suppl):10006. doi:10.1200/ JCO.2019.37.15_suppl.10006

36. Avramis VI, Panosyan EH. Pharmacokinetic/pharmacodynamic relationships of asparaginase formulations: the past, the present and recommendations for the future. Clin Pharmacokinet. 2005;44(4):367-393. doi:10.2165/00003088-200544040-00003

37. Silverman LB, Supko JG, Stevenson KE, et al. Intravenous PEG-asparaginase during remission induction in children and adolescents with newly diagnosed acute lymphoblastic leukemia. Blood. 2010;115(7):1351-1353. doi:10.1182/blood-2009-09-245951

38. Angiolillo AL, Schore RJ, Devidas M, et al. Pharmacokinetic and pharmacodynamic properties of calaspargase pegol Escherichia coli L-asparaginase in the treatment of patients with acute lymphoblastic leukemia: results from Children's Oncology Group Study AALL07P4. J Clin Oncol. 2014;32(34):3874-3882. doi:10.1200/JCO.2014.55.5763

39. Lew G. Space for calaspargase? A new asparaginase for acute lymphoblastic leukemia. Clin Cancer Res. 2020;26(2):325-327. doi:10.1158/1078-0432.CCR-19-2975

40. Pui C-H, Liu Y, Relling MV. How to solve the problem of hypersensitivity to asparaginase? Pediatr Blood Cancer. 2018;65 (3):e26884. doi:10.1002/pbc.26884 
41. Rau RE, Dreyer Z, Choi MR, et al. Outcome of pediatric patients with acute lymphoblastic leukemia/lymphoblastic lymphoma with hypersensitivity to pegaspargase treated with PEGylated Erwinia asparaginase, pegcrisantaspase: a report from the Children's Oncology Group. Pediatr Blood Cancer. 2018;65(3):e26873. doi: $10.1002 / p b c .26873$

42. Zalewska-Szewczyk B, Andrzejewski W, Młynarski W, Jedrychowska-Dańska K, Witas H, Bodalski J. The anti-asparagines antibodies correlate with L-asparagines activity and may affect clinical outcome of childhood acute lymphoblastic leukemia. Leuk Lymphoma. 2007;48(5):931-936. doi:10.1080/ 10428190701292049

43. Rizzari C, Citterio M, Zucchetti M, et al. A pharmacological study on pegylated asparaginase used in front-line treatment of children with acute lymphoblastic leukemia. Haematologica. 2006;91(1):24-31.

44. Stock W, La M, Sanford B, et al. What determines the outcomes for adolescents and young adults with acute lymphoblastic leukemia treated on cooperative group protocols? A comparison of Children's Cancer Group and Cancer and Leukemia Group B studies. Blood. 2008;112(5):1646-1654. doi:10.1182/blood-2008-01-130237

45. Pulte D, Gondos A, Brenner H. Improvement in survival in younger patients with acute lymphoblastic leukemia from the 1980 s to the early 21 st century. Blood. 2009;113(7):1408-1411. doi:10.1182/blood-2008-06-164863

46. Ram R, Wolach O, Vidal L, Gafter-Gvili A, Shpilberg O, Raanani P. Adolescents and young adults with acute lymphoblastic leukemia have a better outcome when treated with pediatric-inspired regimens: systematic review and meta-analysis. Am J Hematol. 2012;87 (5):472-478. doi:10.1002/ajh.23149

47. Gökbuget N, Beck J, Brandt K, et al. Significant Improvement of outcome in Adolescents and Young adults (AYAs) aged 15-35 years with Acute Lymphoblastic Leukemia (ALL) with a pediatric derived adult ALL protocol; results of 1529 AYAs in 2 consecutive trials of the German Multicenter Study Group For Adult ALL (GMALL). Blood. 2013;122(21):839.

48. Boissel N, Auclerc MF, Lhéritier V, et al. Should adolescents with acute lymphoblastic leukemia be treated as old children or young adults? Comparison of the French FRALLE-93 and LALA-94 trials. J Clin Oncol. 2003;21(5):774-780. doi:10.1200/JCO.2003.02.053

49. de Bont JM, Holt B, Dekker AW, et al. Significant difference in outcome for adolescents with acute lymphoblastic leukemia treated on pediatric vs adult protocols in the Netherlands. Leukemia. 2004;18(12):2032-2035. doi:10.1038/sj.leu.2403538

50. Testi AM, Attarbaschi A, Valsecchi MG, et al. Outcome of adolescent patients with acute lymphoblastic leukaemia aged 10-14 years as compared with those aged 15-17 years: long-term results of 1094 patients of the AIEOP-BFM ALL 2000 study. Eur J Cancer. 2019;122:61-71. doi:10.1016/j.ejca.2019.09.004

51. Roberts KG. Genetics and prognosis of ALL in children vs adults. Hematology Am Soc Hematol Educ Program. 2018;2018 (1):137-145. doi:10.1182/asheducation-2018.1.137

52. Hawkins DS, Park JR, Thomson BG, et al. Asparaginase pharmacokinetics after intensive polyethylene glycol-conjugated L-asparaginase therapy for children with relapsed acute lymphoblastic leukemia. Clin Cancer Res. 2004;10(16):5335-5341. doi:10.1158/1078-0432.Ccr-04-0222

53. Parker C, Waters R, Leighton C, et al. Effect of mitoxantrone on outcome of children with first relapse of acute lymphoblastic leukaemia (ALL R3): an open-label randomised trial. Lancet. 2010;376 (9757):2009-2017. doi:10.1016/S0140-6736(10)62002-8

54. Frandsen TL, Heyman M, Abrahamsson J, et al. Complying with the European Clinical Trials directive while surviving the administrative pressure - an alternative approach to toxicity registration in a cancer trial. Eur J Cancer. 2014;50(2):251-259. doi:10.1016/ j.ejca.2013.09.027
55. Schmiegelow K, Attarbaschi A, Barzilai S, et al. Consensus definitions of 14 severe acute toxic effects for childhood lymphoblastic leukaemia treatment: a Delphi consensus. Lancet Oncol. 2016;17(6):e231-e239. doi:10.1016/S1470-2045(16)30035-3

56. Burke MJ, Devidas M, Maloney K, et al. Severe pegaspargase hypersensitivity reaction rates (grade $>/=3$ ) with intravenous infusion vs. intramuscular injection: analysis of 54,280 doses administered to 16,534 patients on children's oncology group (COG) clinical trials. Leuk Lymphoma. 2018;59(7):1624-1633. doi:10.10 80/10428194.2017.1397658

57. Rathod S, Ramsey M, Relling MV, Finkelman FD, Fernandez CA. Hypersensitivity reactions to asparaginase in mice are mediated by anti-asparaginase $\operatorname{IgE}$ and $\operatorname{IgG}$ and the immunoglobulin receptors FceRI and FcyRIII. Haematologica. 2019;104(2):319-329. doi:10.3324/haematol.2018.199448

58. Pieters R, Hunger SP, Boos J, et al. L-asparaginase treatment in acute lymphoblastic leukemia: a focus on Erwinia asparaginase. Cancer. 2011;117(2):238-249. doi:10.1002/cncr.25489

59. Strullu M, Corradini N, Audrain M, et al. Silent hypersensitivity to Escherichia coli asparaginase in children with acute lymphoblastic leukemia. Leuk Lymphoma. 2010;51(8):1464-1472. doi:10.3109/10428194.2010.494316

60. Zalewska-Szewczyk B, Gach A, Wyka K, Bodalski J, Młynarski W. The cross-reactivity of anti-asparaginase antibodies against different L-asparaginase preparations. Clin Exp Med. 2009;9(2):113-116. doi:10.1007/s10238-008-0026-9

61. Woo MH, Hak LJ, Storm MC, et al. Hypersensitivity or development of antibodies to asparaginase does not impact treatment outcome of childhood acute lymphoblastic leukemia. J Clin Oncol. 2000;18(7):1525-1532. doi:10.1200/jco.2000.18.7.1525

62. Müller H-J, Beier R, Löning L, et al. Pharmacokinetics of native Escherichia coli asparaginase (Asparaginase medac) and hypersensitivity reactions in ALL-BFM 95 reinduction treatment. $\mathrm{Br}$ $J$ Haematol. 2001;114(4):794-799. doi:10.1046/j.1365-2141.20 01.03009.x

63. Henriksen LT, Harila-Saari A, Ruud E, et al. PEG-asparaginase allergy in children with acute lymphoblastic leukemia in the NOPHO ALL2008 protocol. Pediatr Blood Cancer. 2015;62 (3):427-433. doi:10.1002/pbc.25319

64. Hojfeldt SG, Wolthers BO, Tulstrup M, et al. Genetic predisposition to PEG-asparaginase hypersensitivity in children treated according to NOPHO ALL2008. Br J Haematol. 2019;184 (3):405-417. doi:10.1111/bjh.15660

65. Tong WH, Pieters R, Kaspers GJ, et al. A prospective study on drug monitoring of PEGasparaginase and Erwinia asparaginase and asparaginase antibodies in pediatric acute lymphoblastic leukemia. Blood. 2014;123(13):2026-2033. doi:10.1182/blood2013-10-534347

66. Kloos RQ, Pieters R, Escherich G, van der Sluis IM. Allergic-like reactions to asparaginase: atypical allergies without asparaginase inactivation. Pediatr Blood Cancer. 2016;63(11):1928-1934. doi:10.1002/pbc.26123

67. Kurtzberg J, Asselin B, Bernstein M, Buchanan GR, Pollock BH, Camitta BM. Polyethylene glycol-conjugated L-asparaginase versus native L-asparaginase in combination with standard agents for children with acute lymphoblastic leukemia in second bone marrow relapse: a Children's Oncology Group Study (POG 8866). J Pediatr Hematol Oncol. 2011;33(8):610-616. doi:10.1097/ MPH.0b013e31822d4d4e

68. Würthwein G, Lanvers-Kaminsky C, Gerss J, et al. Therapeutic drug monitoring of asparaginase: intra-individual variability and predictivity in children with acute lymphoblastic leukemia treated with PEG-Asparaginase in the AIEOP-BFM acute lymphoblastic leukemia 2009 Study. Ther Drug Monit. 2020;42(3):435-444. doi:10.1097/FTD.0000000000000727 
69. Mondelaers V, Ferster A, Uyttebroeck A, et al. Prospective, real-time monitoring of pegylated Escherichia coli and Erwinia asparaginase therapy in childhood acute lymphoblastic leukaemia and non-hodgkin lymphoma in Belgium. Br J Haematol. 2020;190(1):105-114. doi:10.1111/ bjh. 16495

70. Yang Q, Jacobs TM, McCallen JD, et al. Analysis of pre-existing IgG and IgM Antibodies against Polyethylene Glycol (PEG) in the general population. Anal Chem. 2016;88(23):11804-11812. doi:10.1021/acs.analchem.6b03437

71. Salzer WL, Asselin B, Supko JG, et al. Erwinia asparaginase achieves therapeutic activity after pegaspargase allergy: a report from the Children's Oncology Group. Blood. 2013;122 (4):507-514. doi:10.1182/blood-2013-01-480822

72. Verma A, Chen K, Bender C, Gorney N, Leonard W, Barnette P. PEGylated E. coli asparaginase desensitization: an effective and feasible option for pediatric patients with acute lymphoblastic leukemia who have developed hypersensitivity to pegaspargase in the absence of asparaginase Erwinia chrysanthemi availability. Pediatr Hematol Oncol. 2019;36(5):277-286. doi:10.1080/0888 0018.2019.1634778

73. August KJ, Farooki S, Fulbright JM, et al. Desensitization to pegaspargase in children with acute lymphoblastic leukemia and lymphoblastic lymphoma. Pediatr Blood Cancer. 2020;67(1): e28021. doi:10.1002/pbc.28021

74. Marini BL, Brown J, Benitez L, et al. A single-center multidisciplinary approach to managing the global Erwinia asparaginase shortage. Leuk Lymphoma. 2019;60(12):2854-2868. doi:10. 1080/10428194.2019.1608530

75. Raja RA, Schmiegelow K, Frandsen TL. Asparaginase-associated pancreatitis in children. Br J Haematol. 2012;159(1):18-27. doi: $10.1111 /$ bjh. 12016

76. Alvarez OA, Zimmerman G. Pegaspargase-induced pancreatitis. Med Pediatr Oncol. 2000;34(3):200-205. doi:10.1002/(sici)1096$911 \mathrm{x}(200003) 34: 3<200:$ :aid-mpo7>3.0.co;2-t

77. Flores-Calderón J, Exiga-Gonzaléz E, Morán-Villota S, Martín-Trejo J, Yamamoto-Nagano A. Acute pancreatitis in children with acute lymphoblastic leukemia treated with L-asparaginase. J Pediatr Hematol Oncol. 2009;31(10):790-793. doi:10.1097/MPH.0b013e3181b794e8

78. Knoderer HM, Robarge J, Flockhart DA. Predicting asparaginase-associated pancreatitis. Pediatr Blood Cancer. 2007;49(5):634-639. doi:10.1002/pbc.21037

79. Kearney SL, Dahlberg SE, Levy DE, Voss SD, Sallan SE, Silverman LB. Clinical course and outcome in children with acute lymphoblastic leukemia and asparaginase-associated pancreatitis. Pediatr Blood Cancer. 2009;53(2):162-167. doi:10.1002/pbc.22076

80. Samarasinghe S, Dhir S, Slack J, et al. Incidence and outcome of pancreatitis in children and young adults with acute lymphoblastic leukaemia treated on a contemporary protocol, UKALL 2003. Br J Haematol. 2013;162(5):710-713. doi:10.1111/ bjh.12407

81. Rank CU, Wolthers BO, Grell K, et al. Asparaginase-associated pancreatitis in acute lymphoblastic leukemia: results from the NOPHO ALL2008 treatment of patients 1-45 years of age J Clin Oncol. 2020;38(2):145-154. doi:10.1200/JCO.19.02208

82. Wolthers BO, Frandsen TL, Baruchel A, et al. Asparaginaseassociated pancreatitis in childhood acute lymphoblastic leukaemia: an observational Ponte di Legno Toxicity Working Group study. Lancet Oncol. 2017;18(9):1238-1248. doi:10.1016/S1470-2045(17) 30424-2

83. Carpentieri U, Balch MT. Hyperglycemia associated with the therapeutic use of L-asparaginase: possible role of insulin receptors. J Pediatr. 1978;93(5):775-778. doi:10.1016/s00223476(78)81075-0
84. Khan A, Adachi M, Hill JM. Potentiation of diabetogenic effect of L-asparaginase by prednisolone. Horm Metab Res. 1970;2 (5):275-276. doi:10.1055/s-0028-1095058

85. Chan JC, Cockram CS, Critchley JA. Drug-induced disorders of glucose metabolism. Mechanisms and management. Drug Saf. 1996;15(2):135-157. doi:10.2165/00002018-199615020-00005

86. Salzer WL, Devidas M, Shuster JJ, et al. Intensified PEGL-asparaginase and antimetabolite-based therapy for treatment of higher risk precursor-B acute lymphoblastic leukemia: a report from the Children's Oncology Group. J Pediatr Hematol Oncol. 2007;29 (6):369-375. doi:10.1097/MPH.0b013e3180640d54

87. Stock W, Douer D, DeAngelo DJ, et al. Prevention and management of asparaginase/pegasparaginase-associated toxicities in adults and older adolescents: recommendations of an expert panel. Leuk Lymphoma. 2011;52(12):2237-2253. doi:10.3109/ 10428194.2011.596963

88. Bhojwani D, Darbandi R, Pei D, et al. Severe hypertriglyceridaemia during therapy for childhood acute lymphoblastic leukaemia. Eur J Cancer. 2014;50(15):2685-2694. doi:10.1016/j.ejca.2014.06.023

89. Parsons SK, Skapek SX, Neufeld EJ, et al. Asparaginase-associated lipid abnormalities in children with acute lymphoblastic leukemia. Blood. 1997;89(6):1886-1895. doi:10.1182/blood.V89.6.1886

90. Finch ER, Smith CA, Yang W, et al. Asparaginase formulation impacts hypertriglyceridemia during therapy for acute lymphoblastic leukemia. Pediatr Blood Cancer. 2020;67(1):e28040. doi:10.1002/pbc. 28040

91. Tong WH, Pieters R, de Groot-kruseman HA, et al. The toxicity of very prolonged courses of PEGasparaginase or Erwinia asparaginase in relation to asparaginase activity, with a special focus on dyslipidemia. Haematologica. 2014;99(11):1716-1721. doi:10.3324/haematol.2014.109413

92. Cohen H, Bielorai B, Harats D, Toren A, Pinhas-Hamiel O. Conservative treatment of $\mathrm{L}$-asparaginase-associated lipid abnormalities in children with acute lymphoblastic leukemia. Pediatr Blood Cancer. 2010;54(5):703-706. doi:10.1002/pbc.22305

93. Zawitkowska J, Lejman M, Zaucha-Prażmo A, Sekuła N, Greczkowska-Chmiel T, Drabko K. Severe drug-induced hypertriglyceridemia treated with plasmapheresis in children with acute lymphoblastic leukemia. Transfus Apher Sci. 2019;58(5): 634-637. doi:10.1016/j.transci.2019.08.025

94. Sahoo S, Hart J. Histopathological features of L-asparaginaseinduced liver disease. Semin Liver Dis. 2003;23(3):295-299. doi:10.1055/s-2003-42647

95. Toksvang LN, De Pietri S, Nielsen SN, et al. Hepatic sinusoidal obstruction syndrome during maintenance therapy of childhood acute lymphoblastic leukemia is associated with continuous asparaginase therapy and mercaptopurine metabolites. Pediatr Blood Cancer. 2017;64(9). doi:10.1002/pbc.26519

96. Kloos RQH, Pieters R, van den Bos C, van Eijkelenburg NKA, de Jonge R, van der Sluis IM. The effect of asparaginase therapy on methotrexate toxicity and efficacy in children with acute lymphoblastic leukemia. Leuk Lymphoma. 2019;60(12):3002-3010. doi:10.1080/10428194.2019.1613537

97. Schulte RR, Madiwale MV, Flower A, et al. Levocarnitine for asparaginase-induced hepatic injury: a multi-institutional case series and review of the literature. Leuk Lymphoma. 2018;59 (10):2360-2368. doi:10.1080/10428194.2018.1435873

98. Bushman JE, Palmieri D, Whinna HC, Church FC. Insight into the mechanism of asparaginase-induced depletion of antithrombin III in treatment of childhood acute lymphoblastic leukemia. Leuk Res. 2000;24(7):559-565. doi:10.1016/s0145-2126(00)00017-5

99. Caruso V, Iacoviello L, Di Castelnuovo A, et al. Thrombotic complications in childhood acute lymphoblastic leukemia: a meta-analysis of 17 prospective studies comprising 1752 pediatric patients. Blood. 2006;108(7):2216-2222. doi:10.1182/blood2006-04-015511 
100. Nowak-Göttl U, Kenet G, Mitchell LG. Thrombosis in childhood acute lymphoblastic leukaemia: epidemiology, aetiology, diagnosis, prevention and treatment. Best Pract Res Clin Haematol. 2009;22(1):103-114. doi:10.1016/j.beha.2009.01.003

101. Grace RF, Dahlberg SE, Neuberg D, et al. The frequency and management of asparaginase-related thrombosis in paediatric and adult patients with acute lymphoblastic leukaemia treated on Dana-Farber Cancer Institute consortium protocols. $\mathrm{Br}$ $J$ Haematol. 2011;152(4):452-459. doi:10.1111/j.1365-2141.20 10.08524.x

102. Klaassen ILM, Lauw MN, Fiocco M, et al. Venous thromboembolism in a large cohort of children with acute lymphoblastic leukemia: risk factors and effect on prognosis. Res Pract Thromb Haemost. 2019;3(2):234-241. doi:10.1002/rth2.12182

103. Mogensen SS, Harila-Saari A, Mäkitie O, et al. Comparing osteonecrosis clinical phenotype, timing, and risk factors in children and young adults treated for acute lymphoblastic leukemia. Pediatr Blood Cancer. 2018;65(10):e27300. doi:10.1002/pbc.27 300

104. Patel B, Richards SM, Rowe JM, Goldstone AH, Fielding AK. High incidence of avascular necrosis in adolescents with acute lymphoblastic leukaemia: a UKALL XII analysis. Leukemia. 2008;22(2):308-312. doi:10.1038/sj.leu.2405032

105. Liu C, Janke LJ, Kawedia JD, et al. Asparaginase potentiates glucocorticoid-induced osteonecrosis in a mouse model. PLoS One. 2016;11(3):e0151433. doi:10.1371/journal.pone.0151433
106. Te Winkel ML, Appel IM, Pieters R, van den Heuvel-eibrink MM. Impaired dexamethasone-related increase of anticoagulants is associated with the development of osteonecrosis in childhood acute lymphoblastic leukemia. Haematologica. 2008;93(10):15 70-1574. doi:10.3324/haematol.12956

107. Liu C, Kawedia J, Cheng C, et al. Clinical utility and implications of asparaginase antibodies in acute lymphoblastic leukemia. Leukemia. 2012;26(11):2303-2309. doi:10.1038/leu.2012.102

108. Albertsen BK, Grell K, Abrahamsson J, et al. Intermittent versus continuous PEG-Asparaginase to reduce Asparaginase-associated toxicities: a NOPHO ALL2008 Randomized Study. J Clin Oncol. 2019;37(19):1638-1646. doi:10.1200/jco.18.01877

109. Mogensen SS, Schmiegelow K, Grell K, et al. Hyperlipidemia is a risk factor for osteonecrosis in children and young adults with acute lymphoblastic leukemia. Haematologica. 2017;102(5): e175-e178. doi:10.3324/haematol.2016.160507

110. Mattano LA, Devidas M, Friedmann AM, et al. Effect of dexamethasone (DEX) dose modification on osteonecrosis (ON) risk associated with intensified therapies for standard risk acute lymphoblastic leukemia (SR-ALL): a report from the Children's Oncology Group (COG) study AALL0331. J Clin Oncol. 2013;31(15_suppl):10002. doi:10.1200/jco.2013.31.15_suppl.10002
Blood and Lymphatic Cancer: Targets and Therapy

\section{Publish your work in this journal}

Blood and Lymphatic Cancer: Targets and Therapy is an international, peer-reviewed, open access journal focusing on blood and lymphatic cancer research, identification of therapeutic targets and the optimal use of preventative and integrated treatment interventions to achieve improved outcomes, enhanced survival and quality of life for the cancer patient. The manuscript management system is completely online and includes a very quick and fair peer-review system. Visit http://www.dovepress.com/testimonials.php to read real quotes from published authors. 